【电子与信息科学 / Electronics and Information Science】

\title{
混合模型下的雅可比矩阵退火算法优化
}

\author{
王静红 ${ }^{1,2}$, 冯 婵 $^{3}$, 柴变芳 ${ }^{4}$ \\ 1) 河北师范大学计算机与网络空间安全学院，人工智能研究中心，河北石家庄 $050024 ;$ \\ 2) 伊利诺伊大学香槟分校信息科学学院，厄巴纳-香槟市 61801 , 美国; 3) 河北工程技术学院人工智能与大数据学院, \\ 河北石家庄 050091；4）河北地质大学信息工程学院，河北石家庄 050031
}

摘 要: 退火算法可有效发现网络结构的聚类分布情况，但在不同的网络中算法处理结果的准确性有 待提高. 为更精确地识别混合模型网络结构中的数据分布，解决混合模型易陷入局部最大值和收敛等问题， 提出混合模型下雅可比矩阵退火算法. 首先利用逆温度参数 $\beta$ 对模型进行初始化，然后迭代执行计算期望 步骤和最大化步骤 2 个任务; 采用雅可比矩阵计算模型的后验概率, 直至算法达到设定的准确性或收敛. 将建立的雅可比矩阵退火算法与半监督高斯混合模型下的逆模拟退火聚类算法在真实网络上进行对比分析, 实验结果表明, 基于雅可比矩阵的算法在混合网络模型中的准确性更优. 该算法不仅能防止陷入局部最优, 而且能提高分析网络聚类分布的准确性.

关键词: 人工智能理论；复杂网络；混合模型；退火算法；收敛速度；雅可比矩阵；半监督学习

中图分类号：TP181 文献标志码：A doi：10.3724/SP. J.1249.2021.02188

\section{Optimization of Jacobian matrix annealing algorithm based on hybrid model}

\author{
WANG Jinghong ${ }^{1,2}$, FENG Chan ${ }^{3}$, and CHAI Bianfang ${ }^{4}$ \\ 1) Artificial Intelligence Research Center, College of Computer and cyber Sceurity, Hebei Normal University, \\ Shijiazhuang 050024, Hebei Province, P. R. China \\ 2) School of Information Science, University of Illinois at Urbana-Champaign, Urbana, IL 61801, USA \\ 3) College of Artificial Intelligence Big Data, Hebei Institute of Engineering Technology, \\ Shijiazhuang 050091, Hebei Province, P. R. China \\ 4) College of Information Engineering, Hebei GEO University, Shijiazhuang 050031, Hebei Province, P. R. China
}

\begin{abstract}
The annealing algorithm can effectively find the clustering distribution of network structure, but the algorithm accuracy of handling different networks needs to be further improved. In order to identify the data distribution in network structure of mixed model more accurately and solve the problems of local maximum value and convergence of mixed model, the Jacobian matrix annealing algorithm is studied. First, the model is initialized by using the inverse temperature parameter, and then the two tasks of expectation step and maximization step are performed iteratively. The posterior probability of model is calculated based on the Jacobian matrix until the algorithm reaches the set accuracy or meets the convergence condition. The Jacobian matrix annealing algorithm is
\end{abstract}

Received: 2019-09-18; Accepted: 2019-11-11

Foundation: National Science and Technology Major Project of China (2016ZX05046-004) ; Natural Science Foundation of Hebei Province (F2019205303) ; Science and Technology Project of Education Department of Hebei Province (ZD2018023) Corresponding author: Professor WANG Jinghong. E-mail: wangjinghong6301@163.com

Citation: WANG Jinghong, FENG Chan, CHAI Bianfang. Optimization of Jacobian matrix annealing algorithm based on hybrid model $[\mathrm{J}]$. Journal of Shenzhen University Science and Engineering, 2021, 38(2) : 188-193. (in Chinese) 
compared with the inverse simulated annealing clustering algorithm under semi-supervised Gaussian mixture model on the real network, and the experimental results show that the accuracy of Jacobian matrix algorithm in the hybrid network model is better. The proposed algorithm can not only prevent the network from falling into the local optimum, but also improve the accuracy of analyzing network clustering distribution.

Key words: artificial intelligence theory; complex network; hybrid model; annealing algorithm; convergence rate; Jacobian matrix; semi-supervised learning

随着大数据技术的发展，现有网络日渐成为大 规模、动态性强的复杂网络 ${ }^{[1]}$. 互联网社交平台产 生的多是此性质的网络, 而在不确定的复杂网络前 提下有效发现其内部结构是大数据分析的关键任 务 ${ }^{[2]}$. 社区检测 ${ }^{[3]}$ 是网络分析中一类流行的自动识 别技术, 如抑制整个网络的复杂性和发现网络中的 关键节点. 目前虽然已有大量的用于社区网络检测 的方法, 如层次聚类和分割聚类等方法, 但这些方 法仅用于检测链接紧密的子图. 也有一些用于检测 链接相对远距离子图的模型和方法, 这些模型主要 分为随机块模型算法 ${ }^{[4]}$ 、变分贝叶斯推理 ${ }^{[5]}$ 和用于 网络探索的混合模型 ${ }^{[6]}$. 混合模型中期望最大化 (expectation-maximization, EM) 算法的时间复杂度 比随机块模型算法的小. 王圭等 ${ }^{[7]}$ 提出基于逆模拟 退火的半监督高斯混合模型 (anti-annealing semisupervised Gaussian mixture model expectation maximization, ASGMM-EM) 聚类算法, 改善了半监督聚类 中 $\mathrm{EM}$ 算法在计算过程中发生的局部收玫等问题.

本研究基于 ASGMM-EM, 采用雅可比矩阵, 利 用温度参数进行初始化, 提出混合模型下的雅可比 矩阵退火算法, 以期解决网络结构局部最大值和收 玫速度的问题. 分别采用本研究提出的改进后的雅 可比矩阵退火算法和 ASGMM-EM 算法测试其在真 实网络数据集中的检测准确度、调整兰德系数值 (adjusted Rand index, ARI) 和标准互信息值 (normalized mutual information, NMI), 结果表明, 在混 合模型中, 本研究改进后的雅可比矩阵退火算法性 能要优于 ASGMM-EM 算法.

\section{1 半监督高斯混合模型下的逆模拟退 火算法}

确定性逆模拟退火期望最大化 (deterministic anti-annealing EM, DAEM) 算法能有效改善传统 EM 算法关于收玫慢的问题 ${ }^{[8]}$, 并且对传统的无监督类 分布不均衡导致算法性能降低等不足做了优化, 但 该算法没有考虑类太松散或者太密集的半监督数据
集合 ${ }^{[7]}$. 根据这种情况, 王圭等 ${ }^{[7]}$ 提出基于半监督 高斯模型的逆模拟退火 EM ( anti-annealing semisupervised GMM EM, ASGMM-EM)算法.

执行 $\mathrm{E}$ 步 (expectation step) 时计算未标记样本 $\chi_{j}(l+1<j<l+u, l$ 为已标记样本数, $u$ 为未标记 样本数) 属于类 $i(i \in \mathrm{N})$ 的后验概率为

$$
\boldsymbol{\varphi}_{j i}=\frac{\left[\alpha_{i} \cdot N\left(\boldsymbol{\chi}_{j} \mid \boldsymbol{\mu}_{i}, \boldsymbol{\Sigma}_{i}\right)\right]^{\beta}}{\sum_{i=1}^{k}\left[\alpha_{i} \cdot N\left(\boldsymbol{\chi}_{j} \mid \boldsymbol{\mu}_{i}, \boldsymbol{\Sigma}_{i}\right)\right]^{\beta}}
$$

其中, $\chi_{j}$ 为第 $i$ 个高斯分布样本; $\alpha_{i}$ 为第 $i$ 个高斯分 布的混合系数; $\boldsymbol{\mu}_{i}$ 为第 $i$ 个高斯分布的 $n$ 维均值向 量; $\Sigma_{i}$ 为第 $i$ 个高斯分布的 $n \times n$ 的协方差矩阵; $\beta$ 为 逆温度参数; $N\left(\boldsymbol{\chi}_{j} \mid \boldsymbol{\mu}_{i}, \boldsymbol{\Sigma}_{i}\right)$ 为样本 $\boldsymbol{\chi}_{j}$ 由第 $i$ 个高斯分 布生成时的概率密度; $k$ 为高斯分布的个数.

$\mathrm{M}$ 步 (maximization step) 时估计模型参数 $\alpha_{i} 、 \boldsymbol{\mu}_{i}$ 和 $\boldsymbol{\Sigma}_{i}$ 分别为

$$
\begin{aligned}
& \alpha_{i}= \frac{1}{n}\left(\sum_{\chi_{j} \in D_{\mathrm{u}}} \varphi_{j i}+l_{i}\right) \\
& \mu_{i}= \frac{\sum_{\chi_{j} \in D_{\mathrm{u}}} \varphi_{j i} \chi_{j}+\sum_{\left(\chi_{j}, \lambda_{j}\right) \in D_{1} \wedge \lambda_{j}=i} \chi_{j}}{\sum_{\chi_{j} \in D_{\mathrm{u}}} \varphi_{j i}+l_{i}} \\
& \boldsymbol{\Sigma}_{i}=\frac{1}{\sum_{\chi_{j} \in D_{\mathrm{u}}} \varphi_{j i}+l_{i}}\left[\sum_{\chi_{j} \in D_{\mathrm{u}}} \varphi_{j i}\left(\chi_{j}-\mu_{i}\right)\left(\chi_{j}-\mu_{i}\right)^{\mathrm{T}}+\right. \\
&\left.\sum_{\left(\chi_{j}, \lambda_{i}\right) \in D_{1} \wedge \lambda_{j}=i} \varphi_{j i}\left(\chi_{j}-\mu_{i}\right)\left(\chi_{j}-\mu_{i}\right)^{\mathrm{T}}\right]
\end{aligned}
$$

其中, $D_{\mathrm{u}}$ 为未标记样本集合, $D_{\mathrm{u}}=\left\{\boldsymbol{\chi}_{l+1}, \boldsymbol{\chi}_{l+2}, \cdots\right.$, $\left.\chi_{l+u}\right\} ; D_{1}$ 为已标记样本集 $D_{1}=\left\{\left(\chi_{1}, \lambda_{1}\right),\left(\chi_{2}\right.\right.$, $\left.\left.\lambda_{2}\right), \cdots,\left(\chi_{l}, \lambda_{l}\right)\right\} ; \lambda_{j}$ 为已标记样本 $\chi_{j}$ 对应的类标 记; $l_{i}$ 为属于第 $i$ 个高斯混合分布的已标记样本数.

采用 $k$ 均值算法初始化参数, 相似度采用 $n$ 维 空间两点 $X=\left(x_{1}, x_{2}, \cdots, x_{n}\right)$ 和 $Y=\left(y_{1}, y_{2}, \cdots\right.$, $\left.y_{n}\right)$ 的欧式距离 $d(X, Y)=\sqrt{\sum_{i=1}^{n}\left(x_{i}-y_{i}\right)^{2}}$ 计算. 因此, 给定未标记样本 $D_{\mathrm{u}}=\left\{\chi_{l+1}, \chi_{l+2}, \cdots, \chi_{l+u}\right\}$, 
计算未标记样本 $\chi_{j}$ 到第 $i$ 类已标记样本 $\chi_{i}$ 的欧氏距 离 $d\left(\chi_{j}, \chi_{i}\right)$, 则 $\chi_{j}$ 的初始类记为

$$
\phi_{j}^{(0)}=\underset{i \in\{\mathrm{P}, 2, \cdots, k\}}{\arg \min } d_{j i}
$$

ASGMM-EM 算法停止执行条件与文献 $[8]$ 条件 一致.

$$
\frac{\left|L\left(\Theta^{k}\right)-L\left(\Theta^{k-1}\right)\right|}{\left|L\left(\Theta^{k}\right)\right|}<\tau
$$

其中, $\Theta^{k}$ 为第 $k$ 次迭代后的模型参数; $L\left(\Theta^{k}\right)$ 为 $\Theta^{k}$ 的最大似然估计值； $\tau$ 为预设的阈值.

ASGMM-EM 算法伪代码如图 1.

输入: 已标记样本集 $D_{1}=\left\{\left(\chi_{1}, \lambda_{1}\right),\left(\chi_{2}, \lambda_{2}\right), \cdots\right.$, $\left.\left(\chi_{l}, \lambda_{l}\right)\right\} ; \chi_{i}$ 为第 $i$ 个样本, $\lambda_{i}$ 为其类标记; 未标 记样 $D_{\mathrm{u}}=\left\{\chi_{l+1}, \chi_{l+2}, \cdots, \chi_{l+\mu}\right\} ;$ 类的分布个数 $k ;$

输出: 类划分 $C=\left\{C_{1}, C_{2}, \cdots, C_{k}\right\}$

1) 统计已标记样本类的个数 $l$, 并对已标记样本分类;

2）初始化参数 $\Theta=\left[\alpha_{i}, \mu_{i}, \Sigma_{i}\right]$ 和逆温度表 $T=\left\{\beta_{0}\right.$, $\left.\beta_{1}, \cdots, \beta_{m}\right\}$;

3) for $l=0$ to $m$

4) while

5) E 步: 根据式 (1) 计算未标记样本概率 $\varphi_{j i}$;

6) $\mathrm{M}$ 步: 根据 $\mathrm{E}$ 步计算出的未标记样本概率 $\varphi_{j i}$ 和已 标记的样本数 $l_{i}$, 并更新 $\Theta$;

7) if (停止条件)

8) break;

9) end if

10) end while

11) end for

12）根据 $\varphi_{j i}$ 对未标样本进行类划分

图 1 半监督高斯模型的逆模拟退火算法 $\mathbf{E M}$

Fig. 1 Anti-annealing semi-supervised GMM EM, ASGMM-EM

\section{2 混合模型的 EM 算法}

混合模型的结构检测 ${ }^{[6]}$ 在于通过将模型模拟到 实际网络中来观察和推导. 如果存在节点 $i$ 到节点 $j$ 的边缘, 则具有 $N$ 个节点的网络由邻接矩阵 $A$ 表 示, 其元素 $A_{i j}=1$; 否则, $A_{i j}=0$. 设网络节点 $i$ 在 $c$ 个社区的参数设置为三元组 $\left(\left\{g_{i}\right\},\left\{\boldsymbol{\pi}_{r}\right\},\left\{\boldsymbol{\theta}_{r i}\right\}\right)$. 其中, $g_{i}$ 为节点 $i$ 的组分配; $\pi_{r}$ 为组 $r$ 中的节点分布 概率; $\theta_{r i}$ 为从组 $r$ 到节点 $i$ 中有向边的概率. 设 $g$ 为 所有节点节点的组分配集合; $\pi$ 为所有组中的节点
分布概率; $\theta$ 为所有有向边的概率，则从节点 $i$ 到节 点 $j$ 观察到的网络 $A$ 可能性概率为

$$
\begin{aligned}
& P(A, g \mid \pi, \theta)= \\
& P(A \mid g, \pi, \theta) P(g \mid \pi, \theta) P(A \mid \pi, \theta)= \\
& \prod_{i j} P\left(A_{i j} \mid \pi, \theta\right)= \\
& \prod_{i} \sum_{r=1}^{c}\left\{P\left(g_{i}=r \mid \pi\right) \prod_{j} P\left(A_{i j} \mid g_{i}=r, \theta\right)\right\}= \\
& \prod_{i} \sum_{r=1}^{c}\left[\pi_{r} \prod_{j}\left(\theta_{r j}\right)^{A_{i j}}\right]
\end{aligned}
$$

式(7)中的似然对数是

$$
\begin{aligned}
M= & \lg P(A \mid \pi, \theta)= \\
& \sum_{i} \lg \sum_{r=1}^{c}\left[\pi_{r} \prod_{j}\left(\theta_{r j}\right)^{A_{i j}}\right]
\end{aligned}
$$

使用 $\mathrm{EM}$ 算法通过最大化对数似然 $M$ 来估计参 数, 由 Jensen 不等式来算出 $M$ 的下限为

$$
\bar{M}=\sum_{i r} q_{i r}\left(\lg \pi_{r}+\sum_{j} A_{i j} \lg \theta_{r j}\right)-\sum_{i r} q_{i r} \lg q_{i r}
$$

其中, $q_{i r}$ 为节点 $i$ 属于集群 $r$ 的后验概率，满足 $\sum q_{i r}=1$ 归一化条件.

引人拉格朗日乘数 $\lambda$, 则式 $(9)$ 变为

$$
\bar{M}^{\prime}=\bar{M}+\lambda\left(1-\sum_{r} q_{i r}\right)
$$

其中, $\bar{M}^{\prime}$ 的导数为零; $q_{i r}$ 的更新方程为

$$
q_{i r}=\frac{P\left(A_{i}, g_{i}=r \mid \pi, \theta\right)}{P\left(A_{i} \mid \pi, \theta\right)}=\frac{\pi_{r} \prod_{j} \theta_{r j}^{A_{i j}}}{\sum_{s=1}^{N} \pi_{s} \prod_{j} \theta_{s j}^{A_{i j}}}
$$

这里, $\sum_{r} \pi_{r}=1$ 和 $\sum_{j} \theta_{r j}=1$, 则目标函数变为

$$
\mathrm{MM}=\bar{M}+\lambda\left(1-\sum_{r} \pi_{r}\right)+\sum_{j} \beta_{j}\left(1-\sum_{j} \theta_{r j}\right)
$$

其中, $\beta_{j}$ 为第 $j$ 个节点对应的逆温度参数.

令 MM 的导数在式 (7) M 步中为 0 , 则更新方 程为

$$
\begin{aligned}
& \pi_{r}=\frac{1}{N} \sum_{i} q_{i r} \\
& \theta_{r j}=\frac{\sum_{i} A_{i j} q_{i r}}{\sum_{i} d_{i} q_{i r}}
\end{aligned}
$$

其中, $d_{i}$ 为节点 $i$ 的偏度.

3 混合模型的雅可比矩阵退火算法

目前，计算收玫性使用最广的两大工具是 
Hessian 矩阵 ${ }^{[9]}$ 和 Jacobian 矩阵 ${ }^{[10]}$. XIONG 等 ${ }^{[11]}$ 通 过 Hessian 矩阵进行最优测试, 但 Hessian 矩阵太复 杂，无法分析其收玫性，而 CHAOMURILIGE 等 ${ }^{[12]}$ 证明了 Jacobian 矩阵的有效性. 因此, 本研究通过 分析 Jacobian 矩阵并引人对应于反温度的参数 $\beta$, 传 统的 EM 算法是雅可比矩阵退火算法在温度参数 $\beta=1$ 时的特殊情况, 雅可比矩阵退火算法考虑了 $\beta$ 一般情况. 式(11)中 $q$ 的后验概率可以重写为

$$
p_{i r}=\frac{\left(\pi_{r} \prod_{j} \theta_{r j}^{A_{i j}}\right)^{\beta}}{\sum_{s=1}^{N}\left(\pi_{s} \prod_{j} \theta_{s j}^{A_{i j}}\right)^{\beta}}
$$

其中, $\beta$ 为相应的温度参数.

本研究提出的基于雅可比矩阵的退火算法描述 如图 2.

1) 初始 $\beta=\beta_{0}\left(0<\beta_{0} \ll 1\right)$;

2) $\Theta^{(0)}=\left(\pi^{(0)}, \theta^{(0)}\right)$;

3) $T, t=0$

4) 迭代直至收敛，采用式 (15)计算后验概率;

5) 更新 $\pi_{r}^{(t+1)}$ 和 $\theta_{r j}^{(t+1)}$, 并计算 $\Theta^{\text {new }}$;

6) 增加 $\beta$, 若 $\beta \leqslant \beta_{\text {max }}, t=t+1$, 返回步骤 3 , 降低 $\beta$;

7) 迭代至收玫：执行(12); 执行 (13);

8) 若 $\beta \geqslant 1, t=t+1$, 停止运行.

图 2 雅可比矩阵退火算法

Fig. 2 Jacobian matrix annealing algorithm

当雅可比矩阵退火算法算法的收敛速率小于 1 时, 就可得出一个理论上较小的 $\beta_{0}$ 值范围, 范围内 的 $\beta_{0}$ 都符合 DAEM 算法的标准. 根据 $\mathrm{OLVER}^{[13]}$ 的 推论, 如果算法收玫到稳定的固定点 $p^{*}$, 则在点 $p^{*}$ 处的雅可比矩阵的谱半径（或最大特征值）应当 小于 1 .

采用容易计算和具有算法的收玫性质的能力的 雅可比矩阵，在定向网络中计算出雅可比矩阵退火 算法的收玫速率.

雅可比矩阵通项公式为

$f^{\left(p^{*}\right)}=\left.\frac{\partial f^{(\beta)}(p)}{\partial p}\right|_{p=p^{*}}$

由式(15)得到雅可比矩阵. 为了能得到雅可比 矩阵, 首先需要明确雅可比矩阵退火算法算法的参 数映射.

雅可比矩阵退火算法每一轮都经过 $\mathrm{E}$ 步和 $\mathrm{M}$ 步, $F^{(\beta)}$ 表示算法的 $\beta$ 次迭代过程. 其中, $F^{(\beta)}$ 的分
解形式为

$$
F^{(\beta)}=\sum_{t=0}^{N}\left(p_{i r}^{t} ; \boldsymbol{\Theta}_{t}^{\text {new }}\right)^{(\beta)}-\sum_{t=1}^{N}\left(p_{i r}^{t-1} ; \boldsymbol{\Theta}_{t-1}^{\text {new }}\right)^{(\beta)}
$$

采用式 (17) 和式 (18), 雅可比矩阵退火算法 算法的迭代公式可以为映射 $p^{(t+1)}=F^{(\beta)}\left(p^{(t)}\right)$, 则 节点 $i$ 到 $r$ 的映射为

$$
\begin{aligned}
p_{i r}^{(t+1)}= & F^{(\alpha \beta)}\left(p_{i r}^{(t)}\right)= \\
& \frac{\left[\pi_{r}^{(t+1)} \prod_{j}\left(\theta_{r j}^{(t+1)}\right)^{A_{i j}}\right]^{\beta}}{\sum_{s}\left[\pi_{s}^{(t+1)} \prod_{j}\left(\theta_{s j}^{(t+1)}\right)^{A_{i j}}\right]^{\beta}} \\
\pi_{r}^{(t+1)}= & \frac{1}{N} \sum_{i} p_{i r}^{(t)} \\
\theta^{(t+1)_{r j}}= & \frac{\sum_{i} A_{i j} P_{i r}^{(t)}}{\sum_{i} d_{i} P_{i r}^{(t)}}
\end{aligned}
$$

其中, $p^{(t)}(t \in N)$ 是雅可比矩阵退火算法算法的收 玫序列, 该序列会收玫到一个固定点 $p^{*}$, 即 $p^{*}=$ $F^{(\beta)} p^{(*-1)}$. 其中, $p^{(*-1)}$ 为 $p^{*}$ 前一次迭代的收玫序 列. 由于雅可比矩阵在 $\left.\left(\partial F^{(\beta)} p_{i r} / \partial p_{j s}\right)\right|_{p=p^{*}}$ 的点上 的最大特征值不小于 1 , 所以算法不能稳定地收玫 到这些无意义的点上. 雅可比矩阵退火算法有 $\sum_{i=1}^{c} p_{i r}=1$ 的限制, 因此雅可比矩阵 $\left[\left(\partial F^{(\beta)} p_{i r} / \partial p_{j s}\right)_{i j r s}\right]$ 的容积可表示为 $(c-1) \times(c-$ 1) $\times N \times N(i, j \in\{1,2, \cdots, N\}, r, s \in\{1,2, \cdots$, $c-1\}$. 其中, 矩阵的 $(r-1) \times N+i$ 行和 $(s-1) \times$ $N+j$ 列处的元素矩阵可由引理 1 中式 (20) 表示.

引理 1 当 $i=1,2, \cdots, N, r=1,2, \cdots, c-1$, 且 $j=1,2, \cdots, N, s=1,2, \cdots, c-1$ 时, 有

$$
\begin{aligned}
\frac{\partial F_{i r}^{(\beta)}}{\partial p_{j s}}= & \beta \delta_{r s} \times\left(\frac{p_{i r}}{N \pi_{r}}+p_{i r} \sum_{l=1}^{N} \frac{A_{i l}}{\theta_{r l}} \frac{A_{j l}-d_{j} \theta_{r l}}{\sum_{k=1}^{N} d_{k} p_{k r}}\right)- \\
& p_{i r} \beta\left(\frac{p_{i s}}{N \pi_{s}}+p_{i s} \sum_{l=1}^{N} \frac{A_{i l}}{\theta_{s l}} \frac{A_{j l}-d_{j} \theta_{s l}}{\sum_{k=1}^{N} d_{k} p_{k s}}\right)+ \\
& p_{i r} \beta\left(\frac{p_{i c}}{N \pi_{c}}+p_{i c} \sum_{l=1}^{N} \frac{A_{i l}}{\theta_{c l}} \frac{A_{j l}-d_{j} \theta_{c l}}{\sum_{k=1}^{N} d_{k} p_{k c}}\right)
\end{aligned}
$$

其中, $\frac{\partial \theta_{r l}}{\partial p_{j s}}=\frac{A_{j l}-d_{j} \theta_{r l}}{\sum_{k=1}^{N} d_{k} p_{k r}} ; \delta_{r s}$ 为克罗内克 $\delta$ 函数, $\delta_{r s}=\left\{\begin{array}{ll}1, & r=s \\ 0, & r \neq s\end{array} ; k\right.$ 为类的分布个数. 


\section{4 实验及结果分析}

本研究通过实验验证基于雅可比矩阵分析的退 火算法在真实网络上的初始参数数值并对算法的性 能进行分析.

\section{1 基于雅可比矩阵退火算法性能分析}

将优化算法分别在 3 个经典网络数据集 books on politics (以下简称 book) 、adjnoun 和 football 中 进行测试，以此来检测该算法在判断真实网络的准 确性. 由文献 [14] 可知, 当 $\beta=1.15$ 时, 检测混 合模型下雅可比矩阵退火算法的收玫性较好.

首先，调查 book、adjnoun 和 football 3 个真实 网络数据集的 $\beta$ 值对检测网络准确性的影响. 由图 3 可见，在 book 网络数据集中, $\beta$ 的值对雅可比矩 阵退火算法的检测准确性无影响; 在 adjnoun 网络 数据集中, 当 $\beta>0.6$ 时, 雅可比矩阵退火算法的 检测准确性偏低; 在 football 网络数据集中, 准确 度随着 $\beta$ 的增加而降低. 因为 $\beta$ 越小，雅可比矩阵 退火算法越容易收敛到最大值.

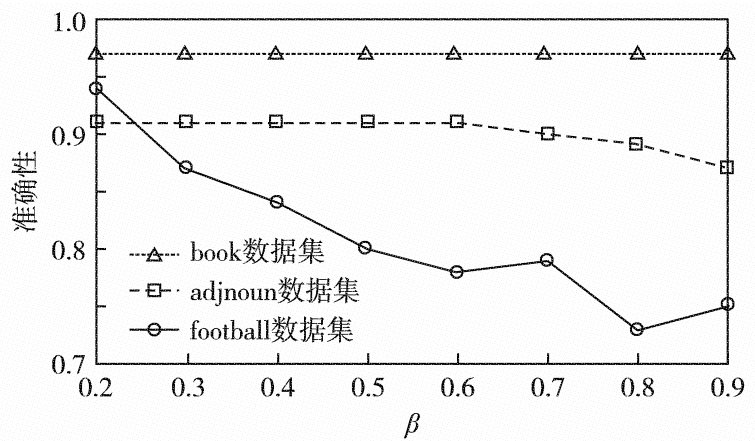

图 3 三个真实网络数据集中不同 $\boldsymbol{\beta}$ 值的 雅可比矩阵退火算法检测准确性对比图

Fig. 3 Accuracy comparison of Jacobian matrix annealing algorithm with different $\beta$ values in three real network data sets

\section{2 对比分析}

为更准确地探索混合模型下雅可比矩阵退火算 法的性能, 本研究设计了混合模型和半监督高斯混 合模型 ( semi-supervised Gaussian mixture model, SGMM)，并从准确性、聚类结果之间相似性度量 (ARI) 和真实聚类的相似度 (NMI) 3 个方面，分别 在不同的 $\beta$ 值下对两个模型进行测试. 图 4 是逆模 拟退火算法和改进的退火算法在 SGMM 中的实验结 果测试对比. 由图 4 可见，逆模拟退火算法在
SGMM 中使用收玫性更强, 准确率更高. 图 5 是逆 模拟退火算法和改进的退火算法在混合模型中的实 验测试结果. 从图 5 可见, 在混合模型中本研究改 进的退火算法的性能更优.

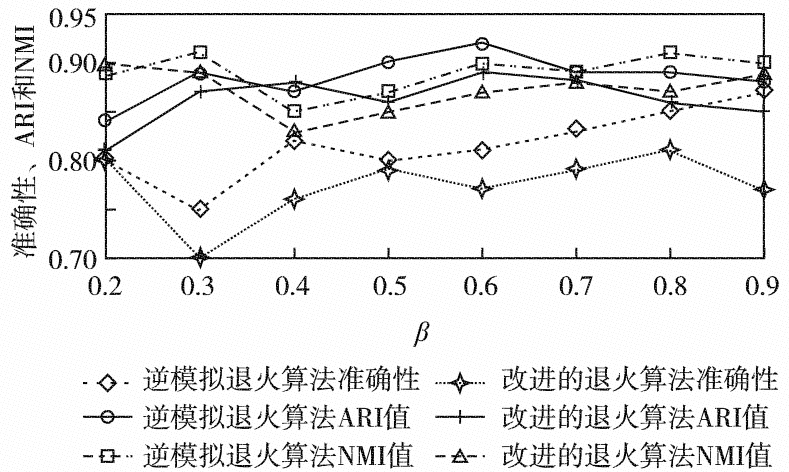

图 4 逆模拟退火算法和改进的退火算法在 SGMM 中 $\beta$ 的准确性、ARI 值、NMI 值对比

Fig. 4 Comparison of the accuracy of $\beta$ 、ARI values and NMI values of the anti-annealing and imprved annealing algorithms in SGMM

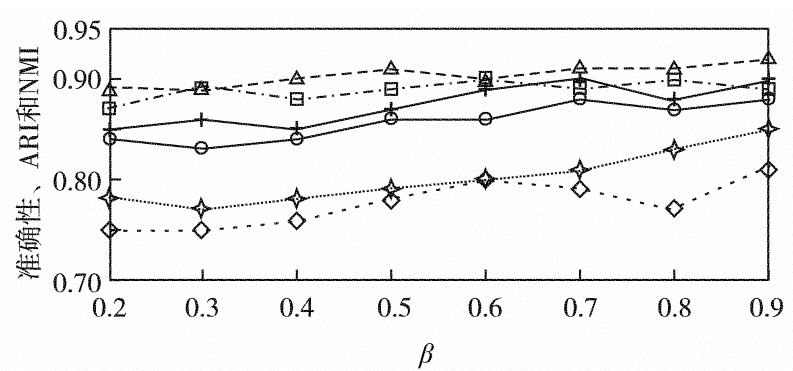

- $\diamond$. 逆模拟退火算法准确性 ……改进的退火算法准确性

- - 逆模拟退火算法ARI值 一 改进的退火算法ARI值 - - - - 逆模拟退火算法NMI值 - A- - 改进的退火算法NMI值

图 5 逆模拟退火算法和改进的退火算法在有向网络混合 模型中 $\beta$ 准确性、ARI 值、NMI 值的对比

Fig. 5 Comparison of the accuracy of $\beta$ 、ARI values and NMI values of the anti-annealing and imprved annealing algorithms in a directed network hybrid model

根据以上对比分析可得, 在本研究中, 混合模 型的退火算法是在基于雅可比矩阵的退火算法上用 来计算收玫速率的，使用确定性抗退火期望最大化 算法可估计混合模型的参数. 根据本研究提供的参 数选择方法的理论下限选择初始参数, 在不小于下 限的情况下，改进的退火算法不会收玫到无意义的 结果，而在不大于上限的情况下，改进的退火算法 可避免收敛到局部最优的结果. 


\section{结 语}

对比逆模拟退火算法和改进的退火算法，逆模 拟退火算法更适用于 SGMM 中，通过实验中数据集 和维度的分析测试，逆模拟退火算法不仅比传统的 高斯混合模型 EM 算法准确率更高，也比改进的退 火算法在 SGMM 中的准确率高. 在逆模拟算法中, 采用的是约束信息基于节点标记的，需由用户先验 提供，并且适合在类不均衡、重叠度密集的情况下 使用. 改进的退火算法是在有向网络下进行研究， 这是因为它更容易扩展到无向网络或加权网络. 本 研究基于雅可比矩阵, 改进了退火算法, 使其更适 合用于清晰的社区结构下的真实网络. 而且，改进 后的退火算法，通过参数选择任务的收玫速率分析 算法以便进行网络探索，更容易在参数的设计上扩 展到其他算法的社区发现.

\section{基金项目：国家科技重大专项资助项目（2016ZX05046-004）；河北 省自然科学基金项目资助项目（F2019205303）；河北省 教育厅科学技术研究资助项目 (ZD2018023) \\ 作者简介: 王静红 $(1967-)$, 河北师范大学教授. 研究方向：机 器学习与数据挖掘、复杂网络. \\ E-mail: wangiinghong6301@163.com \\ 引文: 王静红, 冯 婵, 柴变芳. 混合模型下的雅可比矩阵退 火算法优化 $[\mathrm{J}]$. 深圳大学学报理工版, $2021,38(2)$ ： 188-193.}

\section{参考文献 / References:}

[ 1 ] YIN Nan. A big data analysis method based on modified collaborative filtering recommendation algorithms $[\mathrm{J}]$. Open Physics, 2019, 17(1) : 966-974.

[ 2 ] LIAO C H, CHEN M Y. Building social computing system in big data: from the perspective of social network analysis $[\mathrm{J}]$. Computers in Human Behavior, 2019, 101: 457465.

[ 3 ] FORTUNATO S. Community detection in graphs $[\mathrm{J}]$. Physics Reports, 2010, 486 (3/4/5) : 75-174.

[4] 赵学华, 杨 博, 陈贺昌. 一种高效的随机块模型学 习算法 $[\mathrm{J}]$. 软件学报, 2016, 27(9) : 2248-2264.

ZHAO Xuehua, YANG Bo, CHEN Hechang. Fast learning algorithm for stochastic block model $[\mathrm{J}]$. Journal of Software, 2016, 27(9) : 2248-2264. (in Chinese).

[ 5 ] LATOUCHE P, BIRMELE E, AMBROISE C. Variational Bayesian inference and complexity control for stochastic block models $[\mathrm{J}]$. Statistical Modelling, 2012, 12(1) :
93-115.

[ 6 ] YANG Bo, HE He, HU Xiaoming. Detecting community structure in networks via consensus dynamics and spatial [J]. Physica A: Statistical Mechanics and Its Applications, 2017, 483 (1) : 156-170.

[ 7 ] 王 圭，柴变芳，李文斌，等.一种基于逆模拟退火 和高斯混合模型的半监督聚类算法 $[\mathrm{J}]$. 南京师大学 报自然科学版, 2017，40(3)：67-73.

WANG Yao, CHAI Bianfang, LI Wenbin, et al. A semisupervised clustering algorithm based on anti-annealing and Gaussian mixture model $[\mathrm{J}]$. Journal of Nanjing Normal University Natural Science Edition, 2017, 40(3) : 67-73. (in Chinese).

[ 8 ] NAIM I, GILDEA D. Convergence of the EM algorithm for Gaussian mixtures with unbalanced mixing coefficients [C]// Proceedings of the 29th International Conferences on Machine Learning. Edinburgh, UK: Omnipress, $2012: 1427-1431$.

[ 9 ] KANG S B, PARK J W, Lee I. Accuracy improvement of the most probable point-based dimension reduction method using the hessian matrix $[\mathrm{J}]$. International Journal for Numerical Methods in Engineering, 2017, 111(3) : 203217.

[10 ] RUTKEVICH S B, KURASOV P. A formula for eigenvalues of Jacobi matrices with a reflection symmetry [ $\mathrm{J}]$. Advances in Mathematical Physics, 2018, 2018: 9784091

[11] XIONG Hui, SHANG Pengjian. Weighted multifractal cross-correlation analysis based on Shannon entropy $[\mathrm{J}]$. Communications in Nonlinear Science and Numerical Simulation, 2016, 30(1/2/3) : 268-283.

[12] CHAOMURILIGE, YU Jian, YANG M S. Analysis of parameter selection for Gustafson-Kessel fuzzy clustering using Jacobian matrix $[\mathrm{J}]$. IEEE Transactions on Fuzzy Systems, 2015, 23(6) : 2329-2342.

[13] OLVER P J. Lecture notes on numerical analysis [EB/ OL]. (2008-05-18) [2021-01-05]. http://fourier. eng. hmc. edu/e176/lectures/peter/lno. pdf.

[14] NAIM I, GILDEA D. EM algorithm for Gaussian mixtures with unbalanced mixing coefficient $[\mathrm{C}] / /$ Proceedings of the 29th International Conference on Machine Learning. Edinburgh, UK: ACM Press, 2012: 1427-1431.

【中文责编：英 子; 英文责编：木 柯】 